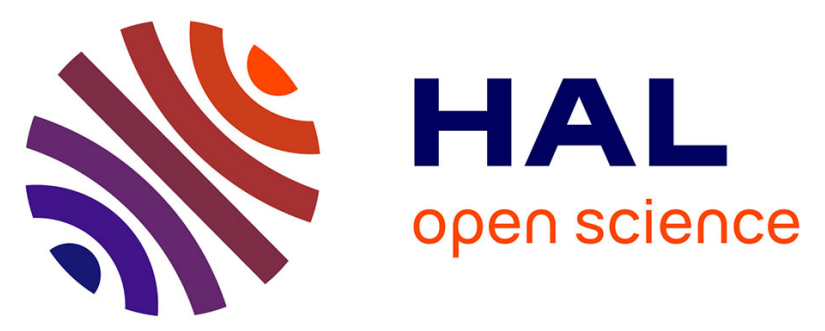

\title{
DICTIONARY LEARNING FOR THE SPARSE MODELLING OF ATRIAL FIBRILLATION IN ECG SIGNALS
}

Boris Mailhé, Rémi Gribonval, Frédéric Bimbot, Mathieu Lemay, Pierre

Vandergheynst, Jean-Marc Vésin

\section{To cite this version:}

Boris Mailhé, Rémi Gribonval, Frédéric Bimbot, Mathieu Lemay, Pierre Vandergheynst, et al.. DICTIONARY LEARNING FOR THE SPARSE MODELLING OF ATRIAL FIBRILLATION IN ECG SIGNALS. ICASSP 2009, Apr 2009, Taipei, Taiwan. pp.465 - 468, 10.1109/ICASSP.2009.4959621. hal-00466973

\section{HAL Id: hal-00466973 https://hal.science/hal-00466973}

Submitted on 25 Mar 2010

HAL is a multi-disciplinary open access archive for the deposit and dissemination of scientific research documents, whether they are published or not. The documents may come from teaching and research institutions in France or abroad, or from public or private research centers.
L'archive ouverte pluridisciplinaire $\mathbf{H A L}$, est destinée au dépôt et à la diffusion de documents scientifiques de niveau recherche, publiés ou non, émanant des établissements d'enseignement et de recherche français ou étrangers, des laboratoires publics ou privés. 


\section{DICTIONARY LEARNING FOR THE SPARSE MODELLING OF ATRIAL FIBRILLATION IN ECG SIGNALS}

\author{
B. Mailhé, R. Gribonval, F. Bimbot \\ Projet METISS, INRIA \\ IRISA, Campus de Beaulieu \\ F-35042 Rennes Cedex, France \\ E-mail: firstname.lastname@irisa.fr
}

\author{
M. Lemay, P. Vandergheynst, J.-M. Vesin \\ Signal Processing Laboratories \\ School of Engineering, EPFL \\ Station 11, CH - 1015 Lausanne, Switzerland \\ E-mail: firstname.lastname@epfl.ch
}

\begin{abstract}
We propose a new method for ventricular cancellation and atrial modelling in the ECG of patients suffering from atrial fibrillation. Our method is based on dictionary learning. It extends both the average beat subtraction and the sparse source separation approaches. Experiments on synthetic data show that this method can almost completely suppress the ventricular activity, but it generates some artifacts. Contrary to other ventricular cancellations methods, our approach also learns a model for the atrial activity.
\end{abstract}

Index Terms - ECG, atrial fibrillation, monochannel source separation, dictionary learning, sparse approximation, K-SVD

\section{INTRODUCTION}

Atrial fibrillation (AF) is the most common type of human arrhythmia and it is responsible for about one third of hospitalizations for arrhythmia problems. AF is more frequent in elderly, as its prevalence doubles with each decade of age, from $0.5 \%$ at ages between $50-59$ years to almost $9 \%$ at ages between $80-89$ years. AF is an important clinical entity because of the increased risk of morbidity and mortality. The most frequent consequences are hemodynamic function impairment (loss of atrial synchronized contraction, irregular and inadequately rapid ventricular rate), atriogenic thromboembolic events and tachycardia induced atrial and ventricular cardiomyopathy. AF diagnosis has been assessed for years by visual inspection of the surface electrocardiogram (ECG). On the ECG, the AF signals are characterized by continuous, apparently disorganized, fibrillatory waves. Due to the much higher amplitude of the electrical ventricular activity (VA) on the surface ECG, isolation of the atrial activity (AA) component in the ECG is crucial for the study of AF.

Some methods used to solve this problem are based on average beat subtraction (ABS), where an average of the ventricular complexes (QRST complexes) is used to subtract VA [1]. Other methods are based on independent component analysis (ICA)[2]. In present ICA based approaches, a major gap is that only statistical priors are considered without taking into account the structural nature of signals. A sparsity-based source separation method has also been explored in [3], which used a simple analytical model to represent both the QRST complexes and the fibrillatory waves.

In this work we propose to learn the dictionaries to be used for sparsity-based source separation. This method is an hybrid of the ABS and sparsity approaches. It can be seen as a way to denoise the templates used in ABS from any atrial interference, or a way to perform sparse source separation with dictionaries that are automatically adapted to the data instead of a rough analytical model.

\section{SPARSE MODELLING AND SOURCE SEPARATION}

\subsection{Multichannel sparse modelling}

A multichannel signal $S$ is a matrix of size $L \times C$, with $L$ the number of samples and $C$ the number of channels. A multichannel dictionary is a set $D$ of multichannel signals called atoms where the signal on each channel has unit euclidean norm. A multichannel dictionary is shift-invariant if it is generated by taking all the possible time shifts $T_{\tau}$ of a limited set $M=\left\{m_{k}\right\}$ of short-time multichannel patterns of size $l \times C$ with unit norm on each channel:

$$
D=\left\{T_{\tau} m_{k}\right\}
$$

A signal $S$ is sparse on a dictionary $D$ if there is a subdictionary $D^{(S)} \subset D$ such that:

$$
\left\{\begin{array}{r}
\left|D^{(s)}\right| \ll|D| \\
\forall c \in[1, C], \Pi_{D_{c}^{(s)}} S_{c}=S_{c}
\end{array}\right.
$$

where $D_{c}$ is the monochannel dictionary containing the components of the atoms of $D$ on channel $c$ and $\Pi_{D} S$ denotes the orthogonal projection of $S$ on $D$. $S$ has a sparse approximation on $D$ if these projections are close to $S$ instead of being equal to it. Finding a sparse decomposition of a signal on 
a dictionary is an NP-complete problem but fast suboptimal algorithms exist, especially in the shift-invariant case. When the dictionary is shift-invariant, this can be seen as a detection problem: the decomposition provides the instants where each pattern occurs in the signal.

\subsection{Source separation using sparsity}

The 12-lead ECGs that are studied here are a mixture of the Ventricular Activity (VA) and the Atrial Activity (AA):

$$
S=V A+A A .
$$

If both sources are known to have sparse approximations on the respective dictionaries $D_{V A}$ and $D_{A A}$, and these dictionaries are mutually incoherent (i.e. a signal that is sparse on one of them cannot be sparse on the other one), then a simple separation algorithm can be used:

- decompose the signal on the union of the dictionaries,

- rebuild each source separately with only the atoms coming from its own dictionary.

Escoda et al. applied this to separate AA and VA components [3]. They used a Gabor dictionary for the AA and Gaussian waveforms for the VA. We propose to use the same separation approach with learnt dictionaries to better match the sources.

This method remains purely morphological. The incoherence between dictionaries only comes from the shapes of the patterns. Contrary to ICA-based approaches [2], the goal here is not to identify the mixing process, but each source contribution to each lead. This approach could also be used for monochannel source separation.

\section{PROCESS}

\subsection{Multichannel dictionary learning}

Dictionary learning is performed using the shift-invariant $K$ $S V D$ algorithm described in [4] that was extended to multichannel signals. This algorithm tries to compute a set of patterns $M$ and a sparse approximation of the signal on the generated dictionary $D^{(S)} \subset D$ that minimizes the approximation error

$$
E=\sum_{c}\left\|S_{c}-\Pi_{D_{c}^{(S)}} S_{c}\right\|_{2}^{2}
$$

It does so by alternating 2 steps:

- decomposition: find a good signal approximation on current dictionary.

- dictionary update: optimize the patterns to minimize the error of the current approximation.
The decomposition step is performed using multichannel Matching Pursuit (MP). MP is a greedy algorithm that iteratively selects the "best" atom $\hat{d}$ from the dictionary and subtracts it from the signal until the residual error has dropped enough. The score function that was used in this work is the $\ell^{2}$ norm of the correlations across the channels:

$$
\hat{d}=\operatorname{argmax}_{d \in D} \sum_{c \in[1, C]}\left\langle S_{c}, d_{c}\right\rangle^{2} .
$$

In the dictionary update step, the problem can be separated into $C$ monochannel problems. If the error on each channel is defined as

$$
E_{c}=\left\|S_{c}-\Pi_{D_{c}^{(S)}} S_{c}\right\|_{2}^{2}
$$

then we have $E=\sum_{c} E_{c}$ with each term $E_{c}$ only depending on the shape of the patterns on channel $c$. Thus the dictionary can be optimized independently on each channel using the monochannel update step. In shift-invariant $K-S V D$, this update step roughly consists in extracting patches from the signal where one pattern was found by the decomposition, then setting their principal component as the new pattern [4].

As there is much inter-patient variability in the VA waveforms, the VA dictionary cannot be learnt on other patients' ECG, rather on the mixture itself. However, trying to learn a dictionary on the mixture does not result in two separate dictionaries with clearly identifiable patterns as VA or AA, rather to a dictionary where each pattern is a mixture itself. This problem was already observed when performing ABS [1]: very few templates (often only 1) can be learnt without having them corrupted with atrial interference on the leads with strong atrial energy (typically V1). We propose an alternate learning scheme to overcome it.

\subsection{Alternate learning scheme}

The main hypothesis behind the ABS approach is that VA and AA are decorrelated during fibrillation, so if one computes an average of all heart beats in the mixture, the AA components will cancel each other out. In the dictionary learning framework, a 1 pattern dictionary learnt from the mixture will contain a clean ventricular pattern.

Our learning scheme consists in extending this idea to atrial modelling. We first learn a 1 pattern VA dictionary on the signal, then subtract it from the mixture to get a rough estimate of AA (this first step is nothing but an ABS). Then, a 1 pattern dictionary learnt from this estimated AA. This pattern is subtracted from the signal and a more accurate (i.e. with more patterns) VA dictionary can be learnt on the obtained VA estimate. We iterate the scheme, each dictionary being learnt on the result of a previous cancellation and the number of patterns keeping increasing. This global algorithm is described in 1 . 


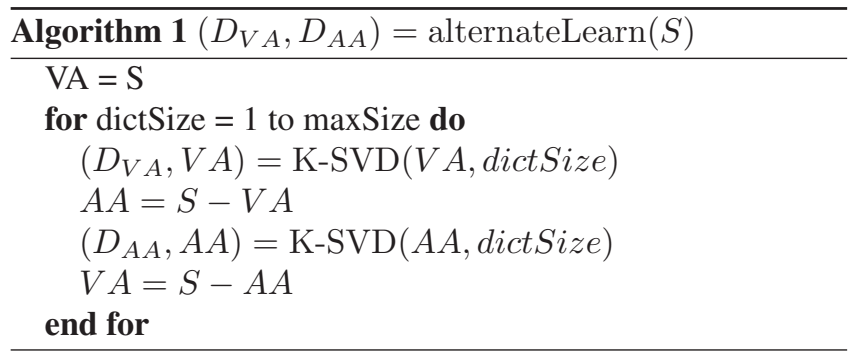

\subsection{Atrial pattern post-processing}

Due to the shape of VA, most of the residual ventricular interference after cancellation tends to be localized in spikes at the QRS wave. As the VA energy is much higher than AA's in the mixture, these spikes can have a high amplitude in the estimates AA even if the VA approximation is good. Then the usual output of dictionary learning on estimated AA is a mixture of these spikes and the normal oscillatory patterns we would like to find. To remove these interferences from the patterns without altering their waveform, a simple spike removal was applied at each iteration of the AA learning algorithm after dictionary update: the highest amplitude spike was compressed down to the level of the second highest one. Negative spikes were processed in the same way.

\subsection{Initialization}

A priori knowledge can be used to find a good initial guess for the patterns. A derivative-based method is applied at the beginning of the algorithm to detect ventricular complexes [5], then at each step the initial VA dictionary is computed using patches taken from the estimated VA at these positions.

The initial AA dictionary is based on the frequency components of the AA. The STFT of the estimated AA is computed before learning the AA dictionary, and the initial dictionary is made of Gabor patterns (i.e. windowed sinusoids) at the frequencies with the most energy in the STFT.

\section{EXPERIMENTAL RESULTS}

\subsection{Database}

A three-dimensional model of the human atria was constructed from magnetic resonance (MR) images, including the openings at the sites of the entries and exits of the vessels as well as at the locations of the valves connecting the atria to the ventricles [6]. In order to create substrates for AF, patchy heterogeneities in action potential duration were introduced by modifying the local membrane properties. Simulated AFs induced by rapid pacing in the left atrium appendage were observed as multiple reentrant wave fronts that propagate and interact in a random fashion over the atrial surface. Nine different simulated AF types, ranging from 11.3 to 23.9 seconds,
Fig. 1. SDR, SIR and SAR (both in $\mathrm{dB}$ ) for both ventricular and auricular estimated activities. As a milestone, the input ratios bewteen VA and AA signals were $12.3 \mathrm{~dB}$ on VR, 11.7 on V1 (the strongest AA) and 17.9 on V4 (the weakest AA).

\begin{tabular}{|cc|ccc|ccc|}
\hline Lead & \multicolumn{3}{|c|}{ dictionaries } & \multicolumn{3}{c|}{ ABS } \\
& & SDR & SIR & SAR & SDR & SIR & SAR \\
\hline VR & VA & 15.6 & 24.1 & 16.7 & 15.1 & 24.3 & 16.1 \\
& AA & 1.2 & 23.0 & 1.4 & -0.5 & 19.2 & 0.5 \\
\hline V1 & VA & 16.4 & 23.3 & 17.7 & 16.8 & 24.6 & 17.9 \\
& AA & 3.0 & 28.4 & 3.1 & 1.5 & 27.9 & 2.5 \\
\hline V4 & VA & 20.3 & 28.9 & 21.3 & 19.8 & 31.5 & 20.2 \\
& AA & -1.4 & 22.2 & -1.3 & -1.9 & 21.1 & -0.7 \\
\hline
\end{tabular}

were created by modifying the pacing protocol and the heterogeneities.

Body surface potentials associated with the AA were computed by using a compartmental torso model constructed from MR images that includes the atria, the ventricles, blood cavities and the lungs [7]. The nine ECG episodes of simulated AF were duplicated to cover five minutes. These nine 5minute ECGs of simulated AA were combined with two different clinical 5-minute standard 12-lead ECGs of patients in sinus rhythm, from which the $\mathrm{P}$ waves were removed. In this manner, 18 realistic simulated 5-minute AF signals sampled at $500 \mathrm{~Hz}$ were created in the standard 12-lead ECG.

\subsection{Results}

The performance measures of our method were the Source to Distortion Ratio (SDR), Source to Interference Ratio (SIR) and Source to Artifact Ratio (SAR) criterions for source separation [8]. The SDR measures the overall distortion between the estimated source and the original one, no matter where this distortion comes from. The SIR measures the amount of the other original sources that is still present in an estimated source. The SAR measures the amount of artifacts that have been introduced in the estimated sources by the separation algorithm itself.

Table 1 shows the SDR, SIR and SAR reached on the VR, V1 and V4 leads, for our method and ABS. The most significant figure for ventricular cancellation is the AA SDR. Here dictionary learning brings a gain on all leads, between 0.5 and $1.5 \mathrm{~dB}$. The VA SIR is lower with dictionaries, which might mean there is still some atrial interference in the ventricular dictionary. However, the finer model also leads to less artifacts in the estimated VA and in all leads except V1, the final result on the VA SDR is positive.

Figure 2 shows an example of the original and estimated activities on lead V1. The estimated AA shown here is the one obtained by cancellation, not the approximation obtained on the AA dictionary:

$$
A A=S-V A .
$$




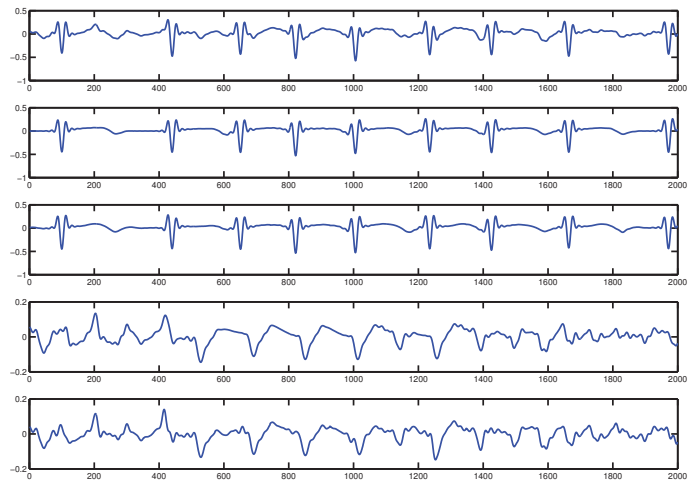

Fig. 2. Sample of the output of the separation algorithm on the V1 lead of a synthetic mixture. From top to bottom: input mixture, ground truth and estimated VA, ground truth and estimated AA.

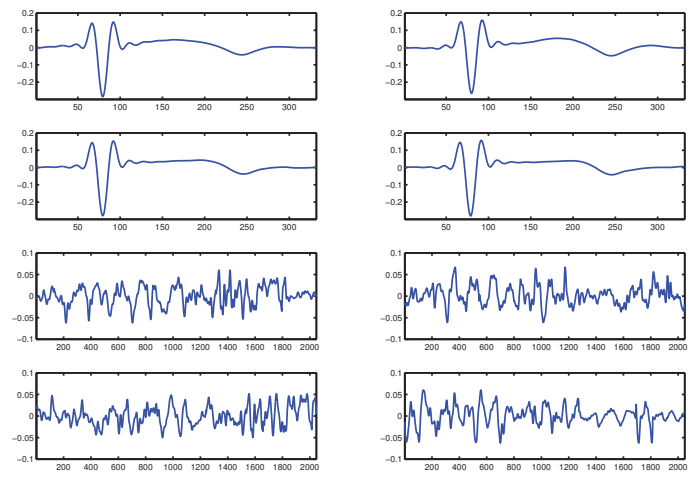

Fig. 3. V1 part of the dictionary on figure 2 mixture. The 4 first patterns form the ventricular dictionary, the latter 4 the atrial one.

The difference is that it also contains the residual that none of the dictionaries could represent. Some high frequency artifacts can be observed during the flat zones of AA.

Figure 3 shows the V1 part of dictionary that was learnt on this mixture. The 4 ventricular patterns do not contain any visible AA. This is an improvement compared to the ABS approach: as V1 is the lead with the most AA energy, it is the one where ventricular patterns are the most likely to contain atrial interferences. It is also probably the main explanation for the high observed SIR.

The different VA patterns are very close to each other. This should not be taken as granted, as it might come from the way our synthetic signals were generated from the VA of patients in sinus rhythm. Clinical data seem to present more diversity in the VA.

\section{CONCLUSION}

We have presented a new source separation method for the visualization of ventricular and atrial activities in ECG signals during atrial fibrillation. Our method is based on sparse modelling of both the ventricular and atrial signals on learnt dictionaries. It extends both the ABS and the sparse separation approaches. It allows the learning of separate atrial and ventricular dictionaries from the mixture signal. It has been tested on synthetic data.

Beyond source separation, the atrial model could prove useful by itself. The dictionary patterns can be seen as a summary of the AA. Instead of cancelling the VA, then look back into the estimated AA for information, some interesting features might be computable directly on the patterns.

\section{REFERENCES}

[1] M. Lemay, V. Jacquemet, A. Forclaz, J.-M. Vesin, and L. Kappenberger, "Spatiotemporal QRST cancellation method using separate QRS and T-waves templates," in Computers in Cardiology 2005. September 2005, IEEE.

[2] F. Castells, J. Igual, J. J. Rieta, C. Sanchez, and J. Millet, "Atrial fibrillation analysis based on ICA including statistical and temporal source information," in ICASSP, 2003, pp. V93-V96.

[3] O. D. Escoda, L. Granai, M. Lemay, J. Molinero Hernandez, P. Vandergheynst, and J.-M. Vesin, "Ventricular and atrial activity estimation through sparse ecg signal decompositions," in International Conference an Acoustics, Speech and Signal Processing (ICASSP'06), 2006.

[4] B. Mailhé, S. Lesage, R. Gribonval, P. Vandergheynst, and F. Bimbot, "Shift-invariant dictionary learning for sparse representations: extending K-SVD," in EUropean SIgnal Processing COnference (EUSIPCO'08), 2008.

[5] Z. Ihara, A. van Oosterom, and R. Hoekama, "Atrial repolarization as observable during the PQ interval," Journal of electrocardiology, vol. 39, no. 3, pp. 290-297, 2006.

[6] V. Jacquemet, M. Lemay, J.-M. Vesin, A. van Oosterom, and L. Kappenberger, "A biophysical model of ECG signals during atrial fibrillation used to evaluate the performance of QRST cancellation algorithms," in Computers in Cardiology 2005. September 2005, IEEE.

[7] A van Oosterom and V Jacquemet, "Genesis of the P wave: atrial signals as generated by the equivalent double layer source model," Europace, p. null, 2005. [in press].

[8] E. Vincent, R. Gribonval, and C. Févotte, "Performance measurement in blind audio source separation," IEEE transactions on audio, speech and language processing, vol. 14, no. 4, pp. 1462-1469, 2006. 